Vol. 01, No. 04, octubre - diciembre 2021, 01-09

\title{
Comparación de dos pruebas de campo para determinar la mastitis subclínica en bovinos en la localidad de Florida - Pomacochas, 2019
}

\section{Comparison of two field tests to determine subclinical mastitis in cattle in the town of Florida - Pomacochas, 2019}

\author{
Carlos U. Escobedo ${ }^{1, a, *}$, Milton J. Trigoso ${ }^{1, b}$, Nilton L. Murga ${ }^{1,2, c}$
}

\footnotetext{
${ }^{1}$ Facultad de Ingeniería Zootecnista, Agronegocios y Biotecnología, Universidad Nacional Toribio Rodríguez de Mendoza de Amazonas, Chachapoyas, Perú.

${ }^{2}$ Instituto de Investigación en Ganaderia y Biotecnología, Universidad Nacional Toribio Rodríguez de Mendoza de Amazonas, Chachapoyas, Perú.
}

a Ing., $\bigotimes_{\text {carlosjalk123478@gmail.com, }}$ iD https://orcid.org/0000-0001-6042-0507

b Mg., $\mathbb{}_{\text {milton.trigoso@untrm.edu.pe, (D) https://orcid.org/0000-0003-4719-5581 }}$

c M.Sc., $\underline{\text { nmurga.fizab@untrm.edu.pe, (iD https://orcid.org/0000-0002-1473-9055 }}$

* Autor de Correspondencia: Tel. +51 931715431

http://dx.doi.org/10.25127/riagrop.20214.716

http://revistas.untrm.edu.pe/index.php/RIAGROP

revista.riagrop@untrm.edu.pe

Recepción: 07 de junio 2021

Aprobación: 12 de agosto 2021

Este trabajo tiene licencia de Creative Commons. Attribution-NonCommercial-ShareAlike $\quad 4.0$ International Public License - CC-BY-NC-SA 4.0

\section{Resumen}

Se evaluaron dos pruebas de campo para determinar la Mastitis Subclinica en bovinos. Se utilizó un total de 268 vacas en producción de leche, entre 1 a 10 partos, en dos sectores San Lorenzo y Miraflores de Levanto. En un sistema extensivo, su alimentación a base de pasturas naturales y cultivadas, un solo ordeño manual, y se consideró desde la segunda semana hasta 5 meses de lactación. Se utilizó el método biológico (Prueba California - Mastitis Test) y el método químico (Prueba de Whiteside), los que permitieron comprobar cuál de las pruebas fue más eficiente para la detección de la mastitis bovina. Los resultados demostraron que el $100 \%$ de prevalencia de mastitis subclínica, según el número de partos, se reporta que el mayor porcentaje de prevalencia se muestra en el 4 parto y según los cuartos mamarios, se reporta que el anterior y posterior derecho reporta el $30 \%$ de prevalencia. Se evidenció un $47 \%$ de efectividad en la prueba Whitside 
y el 53\% en la prueba California Mastitis Test. Esto dificulta la confiabilidad en criadores de ganado con ambas pruebas. Sin embargo, si se tuviera que utilizar una de las dos, sería la California Mastitis Test.

Palabras claves: Prueba california mastitis test, prevalencia, mastitis subclínica, prueba de Whiteside.

\begin{abstract}
Two field tests to determine Subclinical Mastitis in cattle were evaluated. A total of 268 cows were used in milk production from 1 to 10 calvings in two sectors San Lorenzo and Miraflores de Levanto, in an extensive system it is feeding based on natural and cultivated pastures, a single manual milking, and considering from 2 weeks to 5 months of lactation. The biological method (California Mastitis Test) and the chemical method (Whiteside Test) were used to verify, which allowed us to verify which the tests are most effective for detecting bovine mastitis. The results showed that $100 \%$ prevalence of subclinical mastitis was reported, according to the number of births it is reported that the highest prevalence percentage is shown in the 4th birth and according to the fourth mammary it is reported that the anterior and posterior right reports 30\% prevalence. $47 \%$ effectiveness was evidenced in the Whitside test and 53\% in the California Mastitis Test, which hinders the reliability of cattle breeders with both tests; but if you have to use one of the two, the most recommended is the California Mastitis Test.
\end{abstract}

Keywords: California mastitis test, prevalence, mastitis, Whiteside test.

\section{INTRODUCCIÓN}

En la actualidad, la propagación de microorganismos de diferente índole ha sido la causa de infecciones intramamarias en las diversas regiones del mundo. Este hecho ha permitido diversos estudios que han establecido una división de patógenos en ambientales y contagiosos. Todo esto si se tiene en cuenta, además, la interrelación de la epidemia con el grado causante de contagio.

Las unidades de producción son un factor preponderante entorno a la rentabilidad de los productores. Sin embargo, las distorsiones y perturbaciones en la fertilidad, conjuntamente con la mastitis, son uno de los principios que afecta al crecimiento económico de los criadores de ganado, en todas las regiones y partes del planeta. Ambos factores ocasionan pérdidas importantes en la producción y la calidad del producto y con ello disminuye el precio y aumentan los costos de inversión para combatirlo.

Según Zendejas (2020), el incremento permanente de células anatómicas en los infectados por de mastitis genera distorsiones en las composiciones químicas de la leche. La más frecuente de estas es la minimización en calcio, fósforo, grasas y proteínas, que causan incrementos secuenciales en la acumulación de fermento en leche. Mediante este proceso, se comprimen importantes dosis de lactosa y proteína. Todo esto, conjuntamente de constantes alteraciones en la caseína por factores operacionales de enzimas proteolíticas y microorganismos, los mismos que producen bacterias y leucocitos, que afectan al bajo rendimiento y la disminución del periodo en la vida útil del producto. 
López (2017) manifiesta que, para el Consejo Nacional de Estados Unidos, existe un incremento de las inversiones de los productores en los últimos años. Todo esto a partir de que gran parte de las vacas son afectadas por mastitis tienen un valor representativo de un tercio de la población. Así mismo, Canadá y Cuba son las regiones con más pérdidas de 200 dólares por cada vaca a año. Estos valores corroboran lo mencionado líneas atrás, donde el 70 u $80 \%$ del total de pérdidas que tienen relación con la mastitis subclínica, así como en un 20 a $30 \%$ a la denominada mastitis clínica.

El Perú no ha sido ajeno a las pérdidas económicas por causa de la mastitis (clínica y subclínica). Según Velásquez (2017), en su investigación realizada en Huacho San Felipe, el $48 \%$ de los individuos que formaron parte de la población cuentan con mastitis subclínica con prevalencia de células anatómicas. En esta investigación participaron 4364 vacas Holstein divididas en 32 establos. Por ello, en esta investigación, se tuvo como objetivo determinar la prevalencia de mastitis subclínica por cuartos afectados y de acuerdo al número de partos, identificar los grados de infección presentados por mastitis subclínica y comprobar la efectividad de las dos Pruebas de Campo para Determinar la Mastitis Subclínica.

\section{MATERIALES Y MÉTODOS}

\subsection{Lugar de estudio}

El presente estudio se realizó en la cuenca ganadera del distrito de Florida-Pomacochas, ubicada a $2257 \mathrm{~m}$ s. n. m. en la provincia de Bongará, región Amazonas, con una temperatura promedio de $12{ }^{\circ} \mathrm{C}$ y una humedad relativa de $85 \%$.

\subsection{Población y muestra}

La población estuvo constituida por un total de 943 bovinos de las razas Brown Swiss, y Simmental, distribuidos en dos sectores: San Lorenzo (S1) y Miraflores de Levanto (S2).

La muestra fue establecida mediante el muestreo aleatorio simple para poblaciones finitas donde $\mathrm{Z}=95 \%$ y e= $10 \%$, así como, $\mathrm{P}=$ $50 \%$ y $Q=50$. Fueron un total de 268 muestras, como se muestra en la tabla 1.

Tabla 1. Muestra de bovinos por sector según raza Muestra de bovinos por sector Total

\begin{tabular}{lccc} 
Sector & \multicolumn{3}{c}{ según raza } \\
\cline { 2 - 4 } & Brown swiss & Simmental \\
S1 & 66 & 77 & 143 \\
S2 & 62 & 63 & 125 \\
\hline Total & & & 268 \\
\hline
\end{tabular}

\subsection{Material biológico}

Se utilizó un total de 268 vacas productoras de leche entre 1 a 10 partos, criadas extensivamente a estaca o libremente en el potrero, con sus respectivas crías, su alimentación fue a base de pastos cultivados y naturales, con un solo ordeño al día, en presencia de su cría. El tipo de ordeño fue manual, sin distinción del volumen de producción, pero si el tiempo de lactación de 2 semanas hasta 5 meses.

\subsection{Método Biológico (Prueba California Mastitis Test)}

La prueba de CTM se realizó según el horario de ordeño y varió según el caso, tanto por la mañana o por la tarde, en vacas desde las dos 
semanas después del parto hasta los 5 meses de producción. Para ello, se tomó una cantidad de $2 \mathrm{ml}$ de cada uno de los integrantes de la muestra. Para la recolección de la información se tuvo en cuenta una ficha técnica por bovino, que permitió describir e identificar casos negativos y/o positivos según edad, época de lactancia, números de partos, entre otros factores.

Para la prueba California Mastitis Test, se mezcló el reactivo con una cierta cantidad de leche. Es así que este reactivo estuvo compuesto por alquilaril-sulfonato que consiste en un desinfectante aniónico, el mismo que diluye la membrana celular y nuclear con la invariable expulsión de $\mathrm{ADN}$, que al mezclarse con la desinfectante forma una especie de gel. Con ello, cuanto más ADN se muestre, aumentará la densidad del gel, y con ello resulta preciso indicar la existencia de gran cantidad de células anatómicas en la leche, que liberan ese componente (Nacional Mastitis Council, 1996).

\subsection{Método Químico (Prueba de Whiteside)}

Cuando se terminó el trabajo de campo con $\mathrm{CMT}$, se procedió a realizar la prueba química en vacas desde las dos semanas después del parto hasta los cinco meses de producción, donde se mezcló la leche con hidróxido de sodio en un $4 \%$. Este provoca que la leche se congele en pequeñas porciones porque puede ser observado a primera vista. Las porciones aumentarán en tamaño, a partir de la cantidad de células anatómicas que este contenga. Así también para ser un tanto más perceptible, se precisó usar placa acrílica color negro que en la investigación tuvo 4 separadores.

\subsection{Análisis de datos}

Para el análisis de la información se tuvo en cuenta el estadístico de prueba para las pruebas $Z$ que permitió, a través de la estandarización, determinar si se puede rechazar la hipótesis nula según la comparativa con el valor crítico. En consecuencia, se tuvo la prueba de hipótesis referente a la media, con la distribución Normal: Así mismo, al ser $\mathrm{n} \geq 30$, se tuvo en cuenta la prueba de la diferencia entre dos medias con distribución normal, con un nivel de significación del $5 \%$. Para comparar los valores de las dos variables y estimar las mismas, se hizo uso de una prueba estadística correspondiente al coeficiente de correlación de Spearman y se tomó en cuenta el análisis del Método Biológico (Prueba California Mastitis Test) y del Método Químico (Prueba de Whiteside) de cada una de las muestras.

Para medir la eficiencia y al mismo tiempo la prevalencia de mastitis mediante el Método Biológico y el Método Químico (Prueba de Whiteside), se tuvo en cuenta las siguientes fórmulas:

$$
\begin{gathered}
\% \text { de eficiencia }=\frac{\mathrm{N}^{\circ} \text { vacas positivas segun metodo quimico } / \text { biologico }}{\text { Total de vacas evaludas con mastitis }} \times 100 \\
\% \text { de prevalencia }=\frac{\mathrm{N}^{\circ} \text { vacas bovino con mastitis subclínica }}{\text { Total de vacas evaluados según Método Químico/Biológico }} \times 100
\end{gathered}
$$




\section{RESULTADOS Y DISCUSIÓN}

\subsection{Características principales de los bovinos que formaron parte del estudio}

De la población de bovinos evaluados, el $51 \%$ de los bovinos representan a la raza Simmental y solo el 49 \% de Raza Brown Swiss. Se obtuvo que el $25 \%$ son vacas con cuatro partos en mayor medida, seguido por el $24 \%$ con 3 partos, $21 \%$ de dos partos, $14 \%$ de un parto $y$, por último, en menor porcentaje, los bovinos tuvieron 8 y 10 partos con el $1 \%$ como se muestra en la Figura 1. En el sector San Lorenzo se obtuvo mayor cantidad de vacas evaluadas de 3 partos con un $27 \%$, seguido con cuatro partos con el $24 \%$, y en menor porcentaje con 7 y 10 partos con el 1\%. Finalmente, en el sector Miraflores de Levanto, se obtuvo mayor cantidad de vacas evaluadas de 4 partos con un $27 \%$, seguido por las que tuvieron 2 y 3 partos de manera equitativa con el $23 \%$, y en menor medida con el $1 \%$ y $2 \%$ tuvieron 6 y 7 partos. La forma de ordeño del $100 \%$ es manual, en los dos sectores.

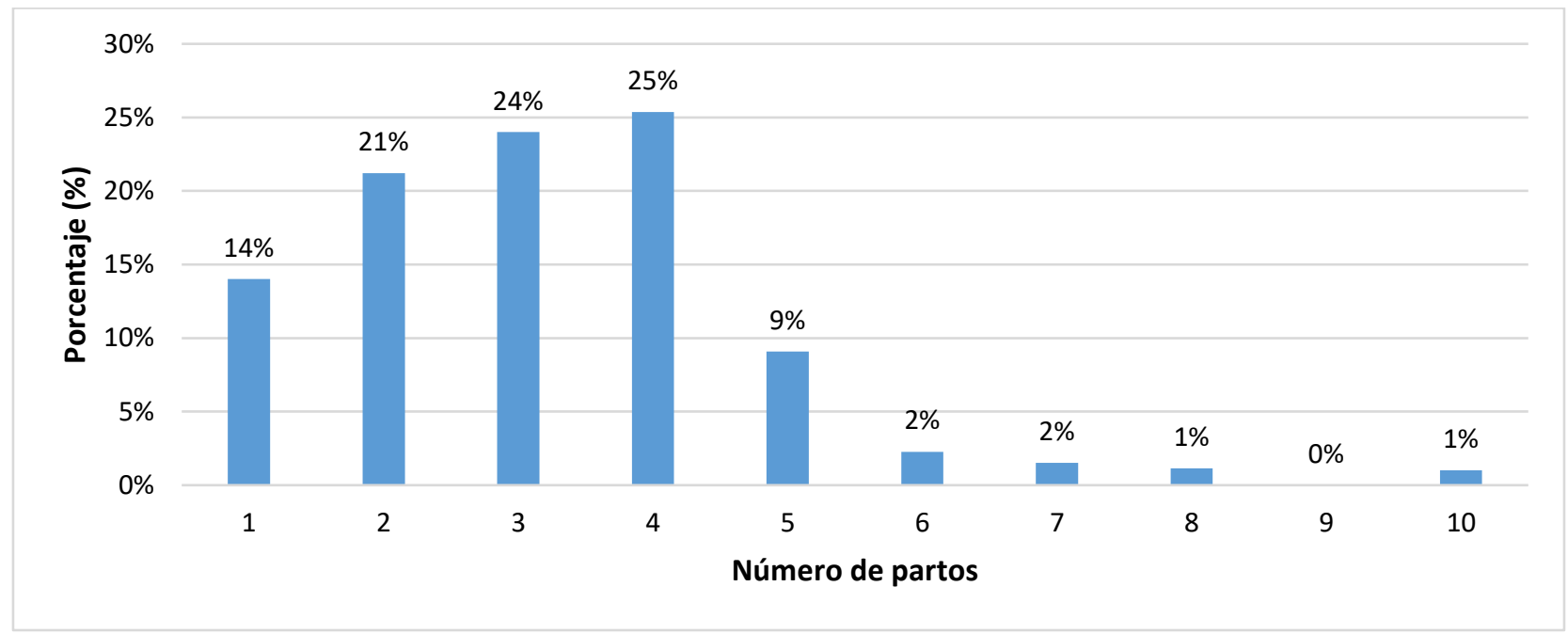

Figura 1. Distribución según el número de partos en la Localidad de Florida-Pomacochas según los sectores: San Lorenzo y Miraflores de Levanto.

3.2. Prevalencia de mastitis subclínica de acuerdo al número de partos y por cuartos afectados

Se evaluó el $27 \%$ del total de bovinos con mastitis subclínica y se obtuvo que del $100 \%$ de las vacas que formaron parte de la población, el $27 \%$ cuenta con mastitis subclínica. Estos resultados fueron superiores a los del estudio realizado por Almeida (2015), acerca de la prevalencia de mastitis bovina mediante la prueba de california mastitis test $\mathrm{e}$ identificación del agente etiológico, que obtuvo un $45 \%$ del área de estudio, el mismo que presentó un alto grado de infección en las vacas lactantes. Adicionalmente, Alva (2013) reportó que el $50 \%$ de prevalencia de mastitis subclínica bovina en el distrito de Calzada, provincia de Moyobamba, un porcentaje elevado con tendencia a incrementarse. Sin embargo, Camacho (2018), en la zona de Alto Imaza, Bongará, reportó que solo el $34.06 \%$ reportó mastitis subclínica. En ese sentido, Mamani et al. 
(2014) reportó valores cercanos a dicha investigación con un a prevalencia de $72.3 \%$ en el distrito de Tamburco, Abancay, Apurímac.

\subsubsection{Prevalencia de mastitis subclínica por cuartos afectados}

La prevalencia de mastitis subclínica por cuartos afectados, en el sector San Lorenzo, estuvo en la zona anterior derecha que presentó un $30 \%$, la zona más afectada, seguido con un $28 \%$ de la zona posterior derecha, en tercer lugar, con un $23 \%$ la parte anterior izquierda y por último con $19 \%$ la zona posterior izquierda. En Miraflores de Levanto, el $29 \%$ de los cuartos afectado fueron en la parte posterior derecha. Así mismo, fueron afectados en un $27 \%$ de la parte posterior derecha. La parte anterior izquierda fue afectada con un $25 \%$ y finalmente la parte posterior izquierda con un $18 \%$. Resultados inferiores al de Gómez-Quispe et al. (2015) reportan una prevalencia de mastitis subclínica con la prueba CMT en los cuartos mamarios individuales varió entre 48.3 y $49.3 \%$ y de 40.0 y $45.5 \%$. Adicionalmente, Camacho, 2018 mediante la prueba CMT reporto valores inferiores en el anterior derecho e izquierdo con una prevalencia de $24.28 \%$ y $20.29 \%$ respectivamente, y superiores en los cuartos mamarios posteriores frente a $18.12 \%$ cuarto posterior derecho y $10.14 \%$ cuarto posterior izquierdo. Sin embargo, Rivera (2014), mediante la prueba CMT, reporta valores inferiores, donde los más afectados fueron los cuartos traseros derechos los delanteros izquierdos, con valores de 19 y $17 \%$, respectivamente.

Según la Prueba de Whiteside en el S1, se puede evidenciar que el $32 \%$ de los bovinos estudiados fueron afectados el parte posterior derecho, seguidos por el parte anterior derecho con un $30 \%$. En tanto, la parte anterior izquierda fue afectada en un $26 \%$ y en menor medida con un $13 \%$ la parte posterior izquierda. En el S2, el 32\% estuvieron afectados en el posterior derecho, seguido del $30 \%$ correspondiente al anterior derecho, en tanto el anterior derecho representa el $23 \%$ y, en menor medida, la parte posterior izquierda con un $16 \%$. Dichos resultados fueron superiores a los reportados por Molleapaza (2001), quien usó la prueba de Whiteside y encontró que el cuarto anterior derecho $1.87 \%$, anterior izquierdo 1.71 $\%$, posterior derecho $1.91 \%$ y posterior izquierdo $1.60 \%$. Adicionalmente. Malaga (2007) reporta valores inferiores, mediante la prueba de Whiteside 0.93, 3.24, 3.70 y $4.63 \%$, para cuartos mamarios anterior derecho, posterior derecho, anterior izquierdo $\mathrm{y}$ posterior izquierdo, respectivamente. Sin embargo, Medina (2003) reporta resultados similares en la evaluación de prevalencia por cuarto mamario mediante la prueba Whiteside de $22.04 \%$ para el anterior izquierdo, $21.38 \%$ para el anterior derecho, $24.67 \%$ para el posterior izquierdo y $23.02 \%$ para posterior derecho.

\subsubsection{Prevalencia de mastitis subclínica por número de partos}

Si se tiene en cuenta la evaluación con la prueba CMT en el sector de Miraflores de Levanto, se obtuvo que, en mayor medida, se encontró de manera positivo con $33 \%$ en los bovinos que tuvieron 4 partos, de 2 partos con un $24 \%$ de manera equitativa. Por último, en menor medida se encontró de manera positivo en las vacas de 6 partos con $3 \%$. En el sector San Lorenzo, en cuanto a positivos para mastitis, con un $28 \%$ se evidenció que las vacas de 4 
partos, y de manera negativa con un $28 \%$ fueron las vacas de 3 partos. Así mismo, en menor medida, las vacas de 10 partos tuvieron $3 \%$ de positivo. Estos resultados fueron similares a los de Santa Cruz (2017), quien, en su estudio, reportó que las vacas con 5 partos presentaron mayor susceptibilidad a la prevalencia de mastitis subclínica en $52.6 \%$ con la prueba CMT y Camacho (2018) menciona que la prevalencia de mastitis según los partos es de mayor número, en orden de mayor a menor para 6 partos $91.67 \%, 8$ partos $85.71 \%, 7$ partos $81.82 \%, 5$ partos $63.89 \%, 4$ partos $37.84 \%$ superior al estudio, 2 partos $26.92 \%$, 3 partos $24.32 \%$. Estos resultados fueron similares al estudio y de 1 parto con $9.52 \%$. En ese sentido, Chasi (2015) menciona que el mayor porcentaje de mastitis obtienen en el tercer parto con un $35.30 \%$.

La prevalencia mediante la prueba T Whiteside, en el sector San Lorenzo, estuvo distribuida de la siguiente manera: en mayor medida con el 29 $\%$ hace referencia a la positividad generalmente en bovinos de 4 partos. Así también, en las vacas de 4 partos se evidenció en mayor medida el lado negativo de la prevalencia de mastitis subclínica con un $26 \%$. En sector de Miraflores de Levanto, se evidenció que las vacas de 4 partos un $30 \%$, en mayor medida, dieron positivo a mastitis subclínica. Además, el $23 \%$ de las vacas evaluadas de 3 partos dieron positivo y en igual medida con $23 \%$ los bovinos de 2 partos dieron negativo. Adicionalmente, las vacas de 10 partos dieron positivo a la mastitis subclínica. Dichos resultados concuerdan con Adiazen y Rodriguez (2007), quien usó la prueba Whiteside y obtuvo un porcentaje de mastitis subclínica en el tercer periodo de lactación (11.20 \%) y según el número de lactaciones la primera tuvo 5 (13.9
$\%)$, que presentaron los mayores porcentajes en la segunda y tercera lactación con 7 (22.2 \%) en la cuarta lactación, 6 (18.4\%) respectivamente.

\subsection{Grados de infección identificados según la mastitis subclínica}

Para identificar los grados de infección de mastitis subclínica, se tuvo en cuenta el número de partos y por cuartos afectados según la evaluación total respecto a los dos tipos de pruebas California Mastitis Test y la prueba Whiteside. A partir del $100 \%$ que dieron positivos, el $57 \%$ fueron de grado 1 , el $26 \%$ de grado 2 y solo el $17 \%$ de grado 3 . Mediante la prueba California Mastitis Test, resultó que del $100 \%$ de los bovinos que dieron positivo a mastitis, en mayor medida, se encuentran en las vacas de 5 partos con un $21 \%$ de grado 1 , en segunda instancia respecto al grado 2, se encuentra las vacas de 4 partos con el $6 \%$. Por último, los bovinos que tuvieron en mayor medida grado 3 fueron las vacas de 8 y 4 partos con un $3 \%$. Con la prueba Whiteside, resultó que, en mayor medida, fueron de grado 1 con 11 $\%$. Los bovinos de 2 partos fueron los que más representa este porcentaje. Así mismo, el $10 \%$ de los que dieron positivo fueron de grado 2 y 3 respectivamente. De esto, las vacas de 4 y 5 partos fueron las más afectadas. Finalmente, en menor medida, los bovinos de 6 y 7 partos tuvieron mastitis de grado1, 2 y 3 , con el $2 \%$ de representatividad.

\subsection{Eficiencia de la prueba Whiteside y California Mastitis Test}

Se tuvo en cuenta la fórmula planteada y se evidencia un $47 \%$ de efectividad en la prueba Whiteside. Esto dificulta la confiabilidad por 
parte de los criadores de ganado. Para la prueba CMT, se evidencia un $53 \%$ de efectividad.

\section{CONCLUSIONES}

La prevalencia de mastitis subclínica está representada por el $27 \%$ del total de bovinos evaluados. La prevalencia de mastitis subclínica por cuartos afectados y por número de partos según la Prueba California Mastitis Test y la Prueba de Whiteside, en mayor medida, el 30\% fueron afectados el anterior derecho y el posterior derecho respectivamente. Así mismo, con el $24 \%$ fue afectado el anterior izquierdo y por último en el posterior izquierdo con un 16 $\%$.

Si se tiene en cuenta los grados de infección, según la evaluación con los dos tipos de pruebas California Mastitis Test y la prueba Whiteside, del $100 \%$ que dieron positivos, el $57 \%$ fue de grado 1 , el $26 \%$ de grado 2 y solo el $17 \%$ de grado 3.

En la eficiencia de las pruebas, se evidenció un $47 \%$ de efectividad en la prueba Whiteside y el $53 \%$ en la prueba California Mastitis Test. Esto dificulta la confiabilidad por parte de los criadores de ganado con ambas pruebas. Pero si se tuviera que utilizar una de las dos, la más recomendable es la California Mastitis Test.

\section{Referencias}

Adianzen, G y Rodriguez, A. (2007). Microorganismos productores de mastitis subclinical bovina y resistencia antibioticos - Ayacucho.

Almeida, D. (2015). Prevalencia de mastitis bovina mediante la prueba de california mastitis test e identificación del agente etiológico, en el centro de acopio de leche en la Comunidad San Pablo Urco, Olmedo - Cayambe - Ecuador, 2014. Quito: Universidad Politecnica Salesiana. Recuperado el 14 de Noviembre de 2019, de
file:///C:/Users/User/Downloads/YT00246_unlocked. pdf

Alva, F. (2013). Prevalencia de mastitis subclínica bovina mediante la prueba de california mastitis test, en el distrito de Calzada-Altomayo, Provincia de Moyobamba 2012. Tesis para optar el título profesional de Médico Veterinario, Facultad de Medicina Veterinaria. Universidad Nacional Pedro Ruiz Gallo, Lambayeque. 45pp.

Camacho, M.C. (2018). Prevalencia de mastitis subclínica mediante la prueba california mastitis test en ganado criollo lechero, distrito de Imaza. Setiembre -diciembre 2017. Tesis UNPRG

Chasi, E.S. (2014). Prevalencia de mastitis bovina mediante la prueba de Califonia Mastitis Test con identificación del agente etiológico, en el centro de acopio de leche de la comunidad de Muyurco, Cayambe - Ecuador, 2014. Obtenido de https://dspace.ups.edu.ec/bitstream/123456789/9839/ 1/UPS-YT00309.pdf

Gómez-Quispe, O; Santivañez-Ballón, C; Arauco- Villar, F; Espezua-Flores, O; Manrique-Meza, F. (2015). Criterios de Interpretación para California Mastitis Test en el Diagnóstico de Mastitis Subclínica en Bovinos. Rev Inv Vet Perú 2015; 26(1): 86-95

López, J. (2017). Levante de novillas de razas lecheras en confinamiento. Revista Universidad de La Salle, 153160.

Malaga, J. (2007). Prevalencia e Incidencia de Mastitis Subclínica en Vacas Brown swiss del CIP Chuquibambilla. Revista de Investigación Universitaria.

Medina, C. (2003). Prevalencia de Mastitis Subclínica bovina en el Valle de Moquegua. Tesis FMVZ-UNA-PUNO.

Molleapaza, R. (2001). Estudio de Prevalencia de Mastitis Subclinica Mediante la Prueba de Whiteside en el Distrito de Taraco, Provincia de Huancané. Tesis FMVZ-UNAPUNO

Rivera Suárez A. (2017). Determinación de la Prevalencia de Mastitis Subclínica en ganado Reyna, Rancho Los Peiranos, Nandaime, Granada. Managua - Nicaragua. Facultad de Ciencia Animal Dpto. de Medicina Veterinaria: Universidad Nacional Agraria, 2014. [Consultado 20 de octubre de 2017]: 79pp. Disponible en : http://repositorio.una.edu.ni/2741/

Santa Cruz, J. (2016). Prevalencia de mastitis subclinica mediante la prueba de California Mastitis Test y relación con el número de partos y los cuartos mamarios afectados en Bovinos (BOS TAURUS) en el distrito de Pulan, provincia de Santa Cruz. [Tesis Bachiller]. Lambayeque - Perú. Facultad de Medicina Veterinaria: Universidad Nacional Pedro Ruiz Gallo, 2017:61pp. 
Velasquez, C. (2010). Factores que influyen en la presentación de mastitis subclínica. Revista Infinitum, 16-21.

Zendejas, E. (29 de Mayo de 2020). Mastitis, problema número 1 en ganado lechero: Manejo, Tratamiento $y$
Control. Bio Zoo S.A de C.V., 1-5. Obtenido de https://www.ganaderia.com/destacado/Mastitis\%2C -problema-numero-1-en-ganado-lechero $\% 3 \mathrm{~A}$ -

Manejo\%2C-Tratamiento-y-

Control\#: :text=La\%20mastitis\%20bovina $\% 2 \mathrm{C} \% 20 \mathrm{ju}$ nto $\% 20$ con,la\%20leche $\% 20 y \% 20$ en $\% 20$ consecuencia 